\title{
Efficiency of two vaccines against Aujeszky's disease (Pseudorabies) Influence of vaccination conditions and of the residual passive immunity on the protection of fattening pigs
}

\author{
P. VANNIER \\ Ministère de l'Agriculture, Direction de la Qualité, Services vétérinaires, \\ Station de Pathologie porcine, B.P. 9, 22440 Ploufragan \\ France
}

The efficiency of live and inactivated virus vaccines against pseudorabies (PR) was compared in fattening pigs. Pigs from vaccinated or unvaccinated sows were vaccinated with one or the other vaccine and challenged at the end of the fattening period. In the first trial, piglets of different ages were vaccinated once. In the second trial, piglets were given twice, half a dose of the inactivated virus vaccine with or without passive immunity. All the pigs were infected with a virulent strain at the end of the fattening period. The particular form of pseudorabies observed in the fattening units was reproduced experimentally. A marked difference appeared between the control group and the groups of pigs vaccinated once or twice with the live and the inactivated virus vaccines. However, a full protection of the pigs was not obtained. The inactivated virus vaccine provided the piglets with a strong passive immunity which interfered with the development of an active immunity. The colostral antibodies were not detected after 5 weeks in piglets born from sows vaccinated with the live strain, whereas they persisted after 10 weeks in those born from sows vaccinated with the inactivated virus vaccine. In all the cases, a double vaccination with half a dosc induced a better immune response than with only one dose of the inactivated virus vaccine.

\section{Piglet health and pathological picture of the respiratory system during the different rearing phases}

\author{
F. MADEC, Marylène KOBISCH \\ Ministère de l'Agriculture, Direction de la Qualité, Services vétérinaires, \\ Station de Pathologie porcine, B.P. 9, 22440 Ploufragan
}

France

\begin{abstract}
Necroscopic examinations were made systematically in piglets randomly selected in conventional commercial units in Brittany (France). The piglets were taken to the laboratory on the day of weaning at the age of 4 weeks (group 1) or about 5 weeks later (group 2). The lesions were less frequent and less severe in group 1 than in group 2 . The frequency of atrophy of the turbinates increased from 20 to $50 \mathrm{p}$. 100 between group 1 and 2 , that of pneumonia from 10 to $24 \mathrm{p} .100$ and that of pleuresy from less than 1 to 8 p. 100. These results were compared with the lesions observed on bacon pigs at the slaughterhouse. It appeared that the frequency of pulmonary lesions increased with age reaching 66 p. 100 for pneumonia and 17 p. 100 for pleuresy, while the number of cases of rhinitis was stable. These pulmonary lesions regressed in the adult sow to 17 and $12 \mathrm{p}$. 100 , respectively for pneumonia and pleuresy, except in the case of severe lesions where after-effects could be observed. The pneumotrophic agents were widely spread in the herds. Bordetella bronchiseptica and Pasteurella multocida were detected in 50 p. 100 of the herds and Haemophilus parasuis in 65 p. 100 of them. These bacteria as well as Mycoplasma hyopneumoniale were found more frequently in the presence than in the absence of lesions. The pathological picture of nasal and pulmonary lesions showed different patterns of development.
\end{abstract}

